Research Article

\title{
Computing the Narumi-Katayama Index and Modified Narumi-Katayama Index of Some Families of Dendrimers and Tetrathiafulvalene
}

\author{
Islam Goli Farkoush, ${ }^{1}$ Mehdi Alaeiyan $\mathbb{D}^{\mathrm{O}},{ }^{2}$ Mohammad Reza Farahani $\mathbb{D}^{\mathrm{D}}{ }^{2}$ \\ and Mohammad Maghasedi ${ }^{1}{ }^{1}$ \\ ${ }^{1}$ Department of Mathematics, Karaj Branch, Islamic Azad University, Karaj, Iran \\ ${ }^{2}$ Department of Mathematics, Iran University of Science and Technology, Narmak, Tehran 16844, Iran
}

Correspondence should be addressed to Mehdi Alaeiyan; alaeiyan@iust.ac.ir

Received 6 May 2021; Revised 26 May 2021; Accepted 5 June 2021; Published 16 June 2021

Academic Editor: Naeem Saleem

Copyright (c) 2021 Islam Goli Farkoush et al. This is an open access article distributed under the Creative Commons Attribution License, which permits unrestricted use, distribution, and reproduction in any medium, provided the original work is properly cited.

\begin{abstract}
A dendrimer is an artificially manufactured or synthesized molecule built up from branched units called monomers. In mathematical chemistry, a particular attention is given to degree-based graph invariant. The Narumi-Katayama index and its modified Narumi-Katayama index of a graph $G$ denoted by NK $(G)$ and $\mathrm{NK}^{*}(G)$ are equal to the product of the degrees of the vertices of $G$. In this paper, we calculate the Narumi-Katayama Index and modified Narumi-Katayama index for some families of dendrimers.
\end{abstract}

\section{Introduction}

A molecular graph is a simple graph related to the structure of a chemical compound. Each vertex of a molecular graph represents an atom of the molecule and its edges to the bonds between atoms. Chemical Graph Theory has an important effect on the development of Chemical Sciences.

In Chemical Science, the multiplicative connectivity indices are used in the analysis of drug molecular structures which are helpful to find out the biological and chemical characteristics of drugs.

Dendrimers are a new class of polymeric materials. They are highly branched, monodisperse macromolecules. The structure of these materials has a great impact on their physical and chemical properties. In chemistry, biochemistry, and nanotechnology, different topological indices are used for modeling physicochemical, pharmacologic, toxicologic, biological, and other properties of chemical compounds. As a result of their unique behavior, dendrimers are suitable for a wide range of biomedical and industrial applications [1].
A molecular graph $G=(V, E)$ with the vertex set $V(G)$ and the edge set $E(G)$ is a graph whose vertices denote atoms and edges denote bonds between the atoms of any underlying chemical structure. The degree of a vertex $v$ of $G$ denoted by $d_{G}$ $(v)$ is the number of edges that are incident to it (for simplicity, $\left.d_{G}(v)=d_{v}\right)$. A topological index Top $(G)$ of graph $G$ is a number with the property, so Top $(H)=$ Top $(G)$ means a graph $H$ is isomorphic with a graph $G$. The idea of topological list originated from work done by Wiener [2]. In [3], Narumi and Katayama considered the product of $d_{\mathrm{v}}$ over all degrees of vertices in $G$ as "simple topological index." Then, the papers, mostly used the name "Narumi-Katayama index" for this index. So, we use from it in this paper, too. In [4-6], authors studied some properties of Narumi-Katayama indices as follows:

$$
\mathrm{NK}(G)=\prod_{u \in V(G)} d_{u}
$$

and the modified of Narumi-Katayama indices as follows:

$$
\mathrm{NK}^{*}(G)=\prod_{u \in V(G)} d_{u^{d_{u}}}
$$


Several articles contributed to determining the topological indices of some families of dendrimer structures and nanostar dendrimers (see [7-15]), porphyrin dendrimers (see [16]), and EThyleneAmidoAmine dendrimers (see $[17,18])$.

In this paper, we compute the Narumi-Katayama index and modified Narumi-Katayama index for some families of dendrimers like $\mathrm{PD}_{1}[n]$ be PAMAM dendrimers with $n$ growth of stages and $n \in N$. For example, the graph $\mathrm{PD}_{2}$ [3] is shown in Figure 1. Another kind of dendrimers, namely, tetrathiafulvalene dendrimer (see Figures 2 and 3), is denoted by $\mathrm{TD}_{2}[n], n \in N \cup$ $\{0\}$. In Figure 3, we can see the graph $\mathrm{TD}_{2}[0]$ and $\mathrm{TD}_{2}[2]$.

\section{Main Results}

In this section, we shall compute the Narumi-Katayama indices and modified Narumi-Katayama index of some families of dendrimers, $\mathrm{PD}_{1}[n], \mathrm{PD}_{2}[n]$, and $\mathrm{TD}_{2}[n]$.

Theorem 1. Let $P D_{1}[n]$ be PAMAM dendrimers with $n$ growth of stages where $n \in N \cup\{0\}$. Then, the Narumi-Katayama index and modified Narumi-Katayama index of $P D_{1}[n]$ are given by

(i) $N K\left(P D_{1}[n]\right)=2^{30 \times 2^{n}-15} \times 3^{9 \times 2^{n}-5}$.

(ii) $N K^{*}\left(P D_{1}[n]\right)=2^{60 \times 2^{n}-30} \times 3^{27 \times 2^{n}-15}$.

Proof. Let $\mathrm{TD}_{1}[n]=\mathrm{G}_{\mathrm{n}}$ where $\mathrm{n} \in \mathrm{NU}\{0\}$. The number of vertices and edges in $G_{n}$ is $48 \times 2^{\mathrm{n}}-23$ and $48 \times 2^{\mathrm{n}}-24$, respectively. The vertex set $V\left(G_{n}\right)$ can be divided into three vertex partitions based on degrees of vertices as $V_{1}, V_{2}$, and $V_{3}$, where $V_{i}=\left\{u \mid u \in V\left(G_{n}\right), \operatorname{deg}(u)=i\right\} ; 1 \leq i \leq 3$. It is easy to see that $\left|V_{1}\left(G_{n}\right)\right|=9 \times 2^{n}-3$; moreover, we have

$$
\left\{\begin{array}{l}
V_{1}\left(G_{n}\right)+2 V_{2}\left(G_{n}\right)+3 V_{3}\left(G_{n}\right)=2 E\left(G_{n}\right) \\
V_{1}\left(G_{n}\right)+V_{2}\left(G_{n}\right)+V_{3}\left(G_{n}\right)=V\left(G_{n}\right)
\end{array} .\right.
$$

Therefore, by solving the above system of equations, the number of vertices in $V_{2}\left(G_{n}\right)$ and $V_{3}\left(G_{n}\right)$ is $30 \times 2^{n}-15$ and $9 \times 2^{n}-5$. Now, by using (1) and (2), we have

$$
\begin{aligned}
& \mathrm{NK}\left(G_{n}\right)=\prod_{u \in V\left(G_{n}\right)} \mathrm{d} u \\
& \text { (i) }=\prod_{u_{1} \in V_{1}\left(G_{n}\right)} \mathrm{d} u_{1} \times \prod_{u_{2} \in V_{2}\left(G_{n}\right)} \mathrm{d} u_{2} \times \prod_{u_{3} \in V_{3}\left(G_{n}\right)} \mathrm{d} u_{3} \\
& =1^{\left|V_{1}\left(G_{n}\right)\right|} \times 2^{\left|V_{2}\left(G_{n}\right)\right|} \times 3^{\left|V_{3}\left(G_{n}\right)\right|} \\
& =1^{1} \times 2^{30 \times 2^{n}-15} \times 3^{9 \times 2^{n}-5} \\
& =2^{30 \times 2^{n}-15} \times 3^{9 \times 2^{n}-5} \text {. } \\
& \mathrm{NK}^{*}\left(G_{n}\right)=\prod_{u \in V\left(G_{n}\right)} \mathrm{d} u^{\mathrm{d} u} \\
& =\prod_{u_{1} \in V_{1}\left(G_{n}\right)} \mathrm{d} u_{1}{ }^{\mathrm{d} u_{1}} \times \prod_{u_{2} \in V_{2}\left(G_{n}\right)} \mathrm{d} u_{2} \mathrm{~d} u_{2} \times \prod_{u_{3} \in V_{3}\left(G_{n}\right)} \mathrm{d} u_{3} \mathrm{~d} u_{3} \\
& =1^{1^{\left|V_{1}\left(G_{n}\right)\right|}} \times 2^{2^{\left|V_{2}\left(G_{n}\right)\right|}} \times 3^{\left.3\right|^{\left|V_{3}\left(G_{n}\right)\right|}} \\
& =2^{2^{30 \times 2^{n}-15}} \times 3^{3^{9 \times 2^{n}-5}} \\
& =2^{60 \times 2^{n}-30} \times 3^{27 \times 2^{n}-15} \text {. }
\end{aligned}
$$

Theorem 2. Let $P D_{2}[n]$ be PAMAM dendrimers with $n$ growth of stages and $n \in N$. Then, the Narumi-Katayama index and its modified of $\mathrm{PD}_{2}[n]$ are given by
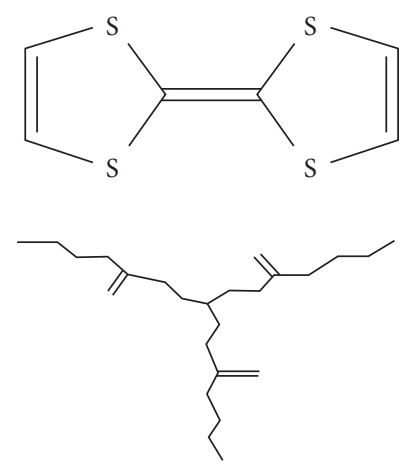

Figure 1: Tetrathiafulvalene $\left(\mathrm{H}_{2} \mathrm{C}_{2} \mathrm{~S}_{2} \mathrm{C}\right)_{2}$ and the core of $\mathrm{PD}_{1}[0]$.

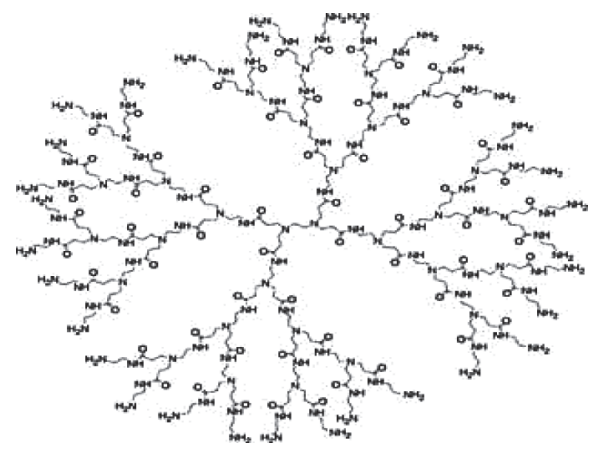

Figure 2: PAMAM dendrimers with 3 growth stages $\mathrm{PD}_{2}$ [3] [18].

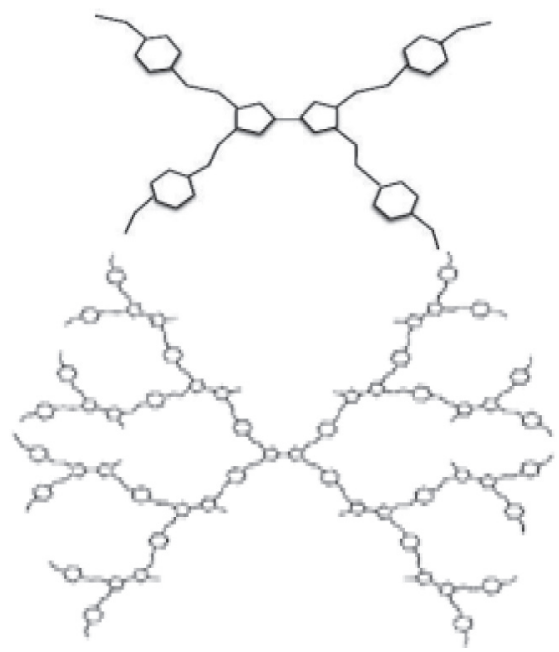

Figure 3: Tetrathiafulvalene dendrimer with 2 growth stages $\mathrm{TD}_{2}$ [0] and $\mathrm{TD}_{2}[2][19-21]$.

(i) $N K\left(P D_{2}[n]\right)=2^{40 \times 2^{n}-4} \times 3^{12 \times 2^{n}+20}$.

(ii) $N K^{*}\left(P D_{2}[n]\right)=2^{80 \times 2^{n}-8} \times 3^{36 \times 2^{n}+60}$.

Proof. This is similar to the proofs of Theorem 1.

Theorem 3. Let $\mathrm{TD}_{2}[n]$ be tetrathiafulvalene dendrimer with $n$ growth of stages and $n \in N \cup\{0\}$. Then, the Narumi-Katayama index and its modified of $\mathrm{TD}_{2}[n]$ are given by

(i) $N K\left(T D_{2}[n]\right)=2^{76 \times 2^{n}-44} \times 3^{40 \times 2^{n}-26}$. 
(ii) $N K^{*}\left(T D_{2}[n]\right)=2^{152 \times 2^{n}-88} \times 3^{120 \times 2^{n}-78}$.

Proof. This is similar to the proofs of Theorems 1 and 2.

Example 1. Consider tetrathiafulvalene dendrimer $\mathrm{TD}_{2}$ $[0]=G_{0}$ where $n \in N \cup\{0\}$ is shown in Figure 3. Theorem 3, $\left|V\left(G_{0}\right)\right|=50$ and $\left|E\left(G_{0}\right)\right|=55$. The vertex partitions $V_{1}\left(G_{0}\right), V_{2}\left(G_{0}\right)$, and $V_{3}\left(G_{0}\right)$ contain, respectively, 4, 32, and 14 vertices. Then,

$$
\begin{aligned}
& \mathrm{NK}\left(G_{0}\right)=\prod_{u \in V\left(G_{0}\right)} \mathrm{d} u \\
\text { (i) } & =\prod_{u_{1} \in V_{1}\left(G_{0}\right)} \mathrm{d} u_{1} \times \prod_{u_{2} \in V_{2}\left(G_{0}\right)} \mathrm{d} u_{2} \times \prod_{u_{3} \in V_{3}\left(G_{0}\right)} \mathrm{d} u_{3} \\
= & 1^{\left|V_{1}\left(G_{0}\right)\right|} \times 2^{\left|V_{2}\left(G_{0}\right)\right|} \times 3^{\left|V_{3}\left(G_{0}\right)\right|} \\
= & 2^{32} \times 3^{14} . \\
& \mathrm{NK}^{*}\left(G_{0}\right)=\prod_{u \in V\left(G_{0}\right)} \mathrm{d} u^{\mathrm{d} u} \\
\text { (ii) } & =\prod_{u_{1} \in V_{1}\left(G_{0}\right)} \mathrm{d} u_{1}{ }^{\mathrm{d} u_{1}} \times \prod_{u_{2} \in V_{2}\left(G_{0}\right)} \mathrm{d} u_{2} \mathrm{~d} u_{2} \times \prod_{u_{3} \in V_{3}\left(G_{0}\right)} \mathrm{d} u_{3} \mathrm{~d} u_{3} \\
= & 1^{\left|V_{1}\left(G_{0}\right)\right|} 2^{2^{\left|V_{2}\left(G_{0}\right)\right|}} \times 3^{3^{\left|V_{3}\left(G_{0}\right)\right|}} \\
= & 2^{64} \times 3^{42} .
\end{aligned}
$$

\section{Conclusions}

In this paper, we determined the Narumi-Katayama index and modified Narumi-Katayama index for some families of dendrimers, namely, PAMAM and tetrathiafulvalene dendrimer. In the future, we are interested to study and compute topological indices of various families of dendrimers or nanostructures, in general.

\section{Data Availability}

No data were used to support this study.

\section{Conflicts of Interest}

The authors declare that they have no conflicts of interest.

\section{References}

[1] B. Klajnert and M. Bryszewska, "Dendrimers: properties and applications," Acta Biochimica Polonica, vol. 48, no. 1, pp. 199-208, 2001.

[2] H. Wiener, "Structural determination of paraffin boiling points," Journal of the American Chemical Society, vol. 69, no. 1, pp. 17-20, 1947.

[3] H. Narumi and M. Katayama, "Simple topological index, A newly devised index characterizingthe topological nature of structural isomers of saturated hydrocarbons," Faculty of Engineering, Hokkaido University, vol. 16, pp. 209-214, 1984.

[4] I. Gutman and M. Ghorbani, "Some properties of the NarumiKatayama index," Applied Mathematics Letters, vol. 25, no. 10, pp. 1435-1438, 2012.

[5] I. Goli Farkoush, M. Alaeiyan, and M. Maghasedi, "On Narumi-Katayama indices of molecular graphs and its modified version," Journal of Discrete Mathematical Sciences and Cryptography, vol. 22, no. 7, pp. 1189-1197, 2019.
[6] I. Goli Farkoush, M. Alaeiyan, M. Alaeiyan, and M. Maghasedi, "Computing the Narumi-Katayama indices and its modified version of some nanostar dendrimers," Eurasian Chemical Communications, vol. 2, no. 7, pp. 771775, 2020.

[7] S. Alikhani and M. A. Iranmanesh, "Chromatic polynomials of some dendrimers," Journal of Computational and Theoretical Nanoscience, vol. 7, no. 11, pp. 2314-2316, 2010.

[8] N. E. Arif, R. Hasni, and S. Alikhani, "Chromatic polynomials of certain families of dendrimers nanostars," Journal of Nanomaterials and Biostructures, vol. 6, pp. 1551-1556, 2011.

[9] A. R. Ashrafi and M. Mirzargar, "PI Szeged, and edge Szeged indices of an infinite family of nanostar dendrimers," Indian Journal of Chemistry, vol. 47, pp. 538-541, 2008.

[10] N. Dorosti, A. Iranmanesh, and M. V. Diudea, "Computing the cluj index of dendrimer nanostars," MATCH Communications in Mathematical and in Computer Chemistry, vol. 62, pp. 389-395, 2009.

[11] M. N. Husin, R. Hasni, and N. E. Arif, "Computation on zagreb polynomial of some families of dendrimers," International Journal of Nanoscience and Nanotechnology, vol. 4, pp. 243-249, 2016.

[12] W. Gao and M. R. Farahani, "The hyper-Zagreb index for an infinite family of nanostar dendrimer," Journal of Discrete Mathematical Sciences and Cryptography, vol. 20, no. 2, pp. 515-523, 2017.

[13] W. Gao, L. Shi, and M. R. Farahani, "Distance-based indices for some families of dendrimer nanostars," IAENG International Journal of Applied Mathematics, vol. 46, no. 2, pp. 168-186, 2016.

[14] M. Imran, S. A. H. Bokhary, S. A. U. h. Bokhary, S. Manzoor, and M. K. Siddiqui, "On molecular topological descriptors of certain families of nanostar dendrimers," Eurasian Chemical Communications, vol. 2, no. 6, pp. 680-687, 2020.

[15] M. Alaeiyan, F. Afzal, M. R. Farahani, and M. A. Rostami, "An exact formulas for the Wiener polarity index of nanostar dendrimers," Journal of Information and Optimization Sciences, vol. 41, no. 4, pp. 933-939, 2020.

[16] A. J. M. Khalaf, A. Javed, M. K. Jamil, M. Alaeiyan, and M. R. Farahani, "Topological properties of four types of porphyrin dendrimers," Proyecciones (Antofagasta), vol. 39, no. 4, pp. 979-993, 2020.

[17] J.-B. Liu, A. Q. Baig, A. Q. Baig et al., "Computation of bond incident degree (BID) indices of complex structures in drugs," Eurasian Chemical Communications, vol. 2, no. 6, pp. 672679, 2020

[18] M. Cancan, S. Ediz, H. Mutee-Ur-Rehman, and D. Afzal, "Mpolynomial and topological indices Poly (EThyleneAmidoAmine) dendrimers," Journal of Information and Optimization Sciences, vol. 41, no. 4, pp. 1117-1131, 2020.

[19] H. Shabani, "Computing vertex PI index of tetrathiafulvalene," Iranian Journal of Mathematical Chemistry, vol. 1, no. 1, pp. 125-130, 2010.

[20] Y.-M. Chu, M. K. Siddiqui, and M. Nasir, "On topological Coindices of polycyclic tetrathiafulvalene and polycyclic oragano silicon," Polycyclic Aromatic Compounds, vol. 16, 2020.

[21] M. Husin, R. Hasni, N. Arif, and M. Imran, "On topological indices of certain families of nanostar dendrimers," Molecules, vol. 21, no. 7, pp. 821-827, 2016. 